\title{
UNITARY STEINBERG GROUP IS CENTRALLY CLOSED
}

\author{
A. LAVRENOV
}

\begin{abstract}
Let $(R, \Lambda)$ be an arbitrary form ring, let $U(2 n, R, \Lambda)$ denote the hyperbolic unitary group, let $E U(2 n, R, \Lambda)$ be its elementary subgroup and $\operatorname{StU}(2 n, R, \Lambda)$ the unitary Steinberg group. It is proved that, if $n \geq 5$ (a natural assumption for similar results), then every central extension of $\operatorname{StU}(2 n, R, \Lambda)$ splits. This results makes it possible to describe the Schur multiplier of the elementary unitary group as the kernel of the natural epimorphism of $\operatorname{StU}(2 n, R, \Lambda)$ onto $E U(2 n, R, \Lambda)$ if it is known that this kernel is included in the center of the unitary Steinberg group. Steinberg's description of relations is employed, which leads to simplest proofs of these results.
\end{abstract}

\section{INTRODUCTION}

In the present paper we prove that the unitary Steinberg group defined by Anthony Bak (see [7, 8, 9, and also [10, 11, 12, 13, 14, 17, 18) is centrally closed.

A similar result for the stable Steinberg group $\operatorname{StU}(R, \Lambda)$, i.e., the direct limit of the sequence

$$
\cdots \rightarrow \operatorname{StU}(2 n, R, \Lambda) \rightarrow \operatorname{StU}(2 n+2, R, \Lambda) \rightarrow \ldots,
$$

was obtained in [7. Actually, by using the same technique it can be proved that the unitary Steinberg groups of finite rank are also centrally closed. However, in [7, 8, 9] Bak used matrix entries rather than roots to classify Steinberg relations among elementary unitary transvections. This leads to 28 relations, and it is quite tiresome to check all detals in such notation. Instead, in this paper we use a simpler approach to the description of relations in terms of roots, which was proposed in [10. This allows us to give an essentially shorter proof than that given by Bak for the stable case.

The results of the present paper imply that if the unitary Steinberg group is a central extension of the elementary unitary group, it is its universal central extension, or, in other words, if the unitary $\mathrm{K}_{2}$ is central in the unitary Steinberg group, then it coincides with the second homology group of the elementary unitary group.

Similar results for Chevalley groups were obtained in [16. The detailed analysis of the linear case can be found in [5, 2. In particular, in [2, Theorem 5.10] it was proved that for $n \geq 5$ the linear Steinberg group $\operatorname{St}(n, R)$ is centrally closed. In the present paper, we follow the same program and prove an analog of this result in the unitary case.

Theorem 1. Let $R$ be an associative unital ring with antiinvolution, let $\Lambda$ be a form parameter, and let $n \geq 5$. Then the unitary Steinberg group $\operatorname{StU}(2 n, R, \Lambda)$ is centrally closed.

By a well-known group theoretical fact, to prove the above theorem it suffices to check the following statement.

2010 Mathematics Subject Classification. Primary 19 C09.

Key words and phrases. Unitary Steinberg group, Schur multiplier, unitary group, form parameter, nonstable $K$-theory. 
Theorem 2. Suppose that $(E, \epsilon)$ is a central extension of the unitary Steinberg group $\mathrm{StU}(2 n, R, \Lambda)$ with $(R, \Lambda)$ a form ring and $n \geq 5$. Then this extension splits, i.e., there exists a group morphism $\sigma: \operatorname{StU}(2 n, R, \Lambda) \rightarrow E$ such that $\epsilon \sigma=\mathrm{id}$.

The paper is organized as follows. The necessary definitions are recalled in $\S \S 1$ and 2, and then the proof of Theorem 1 is given in $\S \S 3-6$.

\section{§1. Unitary Steinberg Group}

In this section we recall the necessary definitions related to form rings and unitary Steinberg groups.

Throughout this paper, $R$ is an arbitrary associative unital ring with antiinvolution $\alpha \mapsto \bar{\alpha}$ on it, i.e., for any $\alpha, \beta \in R$ we have $\overline{\alpha+\beta}=\bar{\alpha}+\bar{\beta}, \overline{\alpha \beta}=\bar{\beta} \bar{\alpha}$, and $\overline{\bar{\alpha}}=\alpha$.

Definition 1. Fix an element $\lambda \in \operatorname{Cent}(R)$ such that $\lambda \bar{\lambda}=1$. Set

$$
\Lambda_{\min }=\{\alpha-\lambda \bar{\alpha} \mid \alpha \in R\}, \quad \Lambda_{\max }=\{\alpha \in R \mid \alpha=-\lambda \bar{\alpha}\} .
$$

A form parameter $\Lambda$ is an additive subgroup of $R$ such that

(1) $\Lambda_{\min } \subseteq \Lambda \subseteq \Lambda_{\max }$,

(2) $\alpha \Lambda \bar{\alpha} \subseteq \Lambda$ for all $\alpha \in R$.

The pair $(R, \Lambda)$ is called a form ring.

In the sequel $\lambda$ denotes a fixed central element, and $\Lambda_{\min }, \Lambda_{\max }$, and $\Lambda$ are the minimal, the maximal, and an arbitrary form parameters with symmetry $\lambda$, respectively.

To define the unitary group, consider a free right $R$-module $V \cong R^{2 n}$. The elements of $V$ may be thought of as columns of height $2 n$ with components in $R$. In particular, let $e_{i}$ be a column whose $i$ th coordinate is 1 while all other coordinates are zeros. Below we index this base as follows: $e_{1}, \ldots, e_{n}, e_{-n}, \ldots, e_{-1}$. We denote the set $\{1, \ldots, n$, $-n, \ldots,-1\}$ of indices by $\Omega,\{1, \ldots, n\}$ by $\Omega_{+}$, and $\{-n, \ldots,-1\}$ by $\Omega_{-}$. For $i, j \in \Omega$, let $e_{i j}$ be the standard matrix unit, i.e., the matrix of size $2 n \times 2 n$ that has 1 on the intersection of the $i$ th row and $j$ th column and zeros elsewhere. Let $e$ be the identity matrix, i.e., the matrix that has 1's along the principal diagonal and zeros elsewhere. Also, let $p_{n}$ be the matrix of size $n \times n$ that has 1's along the skew diagonal and zeros elsewhere. Throughout this paper we assume that $n \geq 3$.

Definition 2. Consider the sesquilinear form $f$ on $V$ that has the Gram matrix $\left(\begin{array}{cc}0 & p_{n} \\ 0 & 0\end{array}\right)$ with respect to the base $e_{1}, \ldots, e_{-1}$. Using this form, define an even $\lambda$-Hermitian form $h=f+\lambda \bar{f}$, where $\bar{f}(u, v)=\overline{f(v, u)}$, and a $\Lambda$-quadratic form $q: V \rightarrow R / \Lambda$, by $q(u)=$ $f(u, u) \bmod \Lambda$.

By the unitary group we mean the group

$$
\mathrm{U}(2 n, R, \Lambda)=\{g \in \mathrm{GL}(2 n, R) \mid \forall u, v \in V h(g u, g v)=h(u, v), q(g u)=q(u)\} .
$$

In other words, the unitary group consists of all elements in $\operatorname{GL}(V) \cong \operatorname{GL}(2 n, R)$ that preserve the $\lambda$-Hermitian form $h$ and the $\Lambda$-quadratic form $q$.

For an element $i$ in $\Omega$, put $\varepsilon(i)=1$ if $i \in \Omega_{+}$and $\varepsilon(i)=-1$ if $i \in \Omega_{-}$.

Definition 3. For $i \in \Omega, j \in \Omega \backslash\{ \pm j\}$, and $\zeta \in R$, define

$$
T_{i j}(\zeta)=e+\zeta e_{i j}-\lambda^{(\varepsilon(j)-\varepsilon(i)) / 2} \bar{\zeta} e_{-j,-i} .
$$

Similarly, for $i \in \Omega$ and $\alpha \in \lambda^{-(\varepsilon(i)+1) / 2} \Lambda$, we define

$$
T_{i,-i}(\alpha)=e+\alpha e_{i,-i} .
$$


These elements will be referred to as the "elementary unitary transvections". The subgroup of the unitary group generated by all elementary unitary transvections is called the elementary unitary group and is denoted by $\mathrm{EU}(2 n, R, \Lambda)$.

It is easy to check that the following relations are fulfilled for the elementary unitary transvections.

Lemma 1. Suppose that $i, h \in \Omega, j \in \Omega \backslash\{i\}, k \in \Omega \backslash\{h\}, \alpha \in \lambda^{-(\varepsilon(i)+1) / 2} \Lambda$, $\zeta, \xi, \eta \in R$. Also, suppose that either $i \neq j$ or $\zeta, \xi \in \lambda^{-(\varepsilon(i)+1) / 2} \Lambda$, and either $h \neq k$ or $\eta \in \lambda^{-(\varepsilon(h)+1) / 2} \Lambda$. Then:

$$
\begin{aligned}
& T_{i j}(\zeta)=T_{-j,-i}\left(-\lambda^{(\varepsilon(j)-\varepsilon(i)) / 2} \bar{\zeta}\right) \\
& T_{i j}(\zeta) T_{i j}(\xi)=T_{i j}(\zeta+\xi)
\end{aligned}
$$

if $h \notin\{j,-i\}, k \notin\{i,-j\}$, then

$$
\left[T_{i j}(\zeta), T_{h k}(\eta)\right]=e ;
$$

if $i, h \notin\{ \pm j\}$ and $i \notin\{ \pm h\}$, then

$$
\left[T_{i j}(\zeta), T_{j h}(\xi)\right]=T_{i h}(\zeta \xi)
$$

if $i \notin\{ \pm j\}$, then

$$
\left[T_{i j}(\zeta), T_{j,-i}(\xi)\right]=T_{i,-i}\left(\zeta \xi-\lambda^{-\varepsilon(i)} \bar{\xi} \bar{\zeta}\right)
$$

if $i \notin\{ \pm j\}$, then

$$
\left[T_{i,-i}(\alpha), T_{-i, j}(\zeta)\right]=T_{i j}(\alpha \zeta) T_{-j, j}\left(-\lambda^{(\varepsilon(j)-\varepsilon(-i)) / 2} \bar{\zeta} \alpha \zeta\right) .
$$

Relations R1-R6 are called the unitary Steinberg relations. These relations define the unitary Steinberg group.

Definition 4. The unitary Steinberg group $\operatorname{StU}(2 n, R, \lambda)$ is the group defined by the generators $X_{i j}(\zeta)$, where $i \in \Omega, j \in \Omega \backslash\{i\}, \zeta \in R$, such that either $i \neq-j$ or $\zeta \in \lambda^{-(\varepsilon(i)+1) / 2} \Lambda$, and relations $\mathrm{R} 1-\mathrm{R} 6$ with $X_{i j}(\zeta)$ in place of $T_{i j}(\zeta)$ :

$$
\mathrm{StU}(2 n, R, \Lambda)=\left\langle X_{i j}(\zeta) \mid \mathrm{R} 1-\mathrm{R} 6\right\rangle .
$$

We denote by $\varpi$ the natural group epimorphism from the unitary Steinberg group to the elementary unitary group, sending the generator $X_{i j}(\zeta)$ to the elementary transvection $T_{i j}(\zeta)$. Denote $\mathrm{K}_{2} \mathrm{U}(2 n, R, \Lambda)=\operatorname{Ker} \varpi$. In the present paper we prove that if

$$
\mathrm{K}_{2} \mathrm{U}(2 n, R, \Lambda) \subseteq \operatorname{Cent}(\operatorname{StU}(2 n, R, \Lambda)),
$$

then $\mathrm{K}_{2} \mathrm{U}(2 n, R, \Lambda)$ coincides with the second homology group of the elementary unitary group:

$$
\mathrm{K}_{2} \mathrm{U}(2 n, R, \Lambda)=\mathrm{H}_{2}(\mathrm{EU}(2 n, R, \Lambda), \mathbb{Z}) .
$$

\section{$\S 2$. Central extensions}

The notion of a central extension was introduced by Schur for finite groups. This theory for arbitrary groups can be found in Steinberg's book [5], and his definitions are equivalent to those by Schur in the case of finite groups. These results can be found also in 2 .

Definition 5. Consider a short exact sequence of groups

$$
1 \longrightarrow C \longrightarrow E \stackrel{\epsilon \longrightarrow}{\longrightarrow} G \longrightarrow 1
$$

such that $C \subseteq \operatorname{Cent}(E)$. We shall call $(E, \epsilon)$ the central extension of the group $G$. 
For a fixed group $G$, an initial object in the category of its central extensions is called the universal central extension of $G$.

Definition 6. A central extension $(U, \pi)$ of a group $G$ is called its universal central extension if for any central extension $(E, \epsilon)$ of this group there is a unique group morphism $\psi: U \rightarrow E$ making the diagran 1

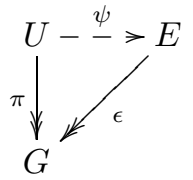

commutative.

Clearly, the universal central extension is unique if it exists.

Lemma 2. If $(U, \pi),\left(U^{\prime}, \pi^{\prime}\right)$ are two universal central extensions of a group $G$, then there exists an isomorphism $\varphi: U \rightarrow U^{\prime}$ such that the diagram

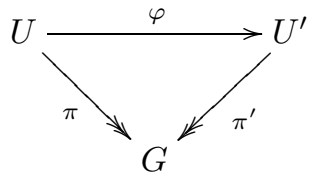

is commutative.

However, not every group admits a universal central extension.

Lemma 3. A group $G$ admits a universal central extension if and only if it is perfect (i.e., coincides with its commutator subgroup $[G, G]$ ).

This lemma is proved, for example, in [2, Theorem 5.7].

Definition 7. Let $(U, \pi)$ be a universal central extension of a group $G$. Then $\operatorname{Ker} \pi$ is called the Schur multiplier of $G$.

Hopf defined the second homology group $\mathrm{H}_{2}(G ; \mathbb{Z})$ of a group $G$ precisely as its Schur multiplier. For the usual definition of the $\mathrm{H}_{2}$, in [2, Corollary 5.8] is was shown that it coincides with a Schur multiplier of a perfect group.

We need a little more notation.

Definition 8. Consider a central extension $(E, \epsilon)$ of a group $G$ and consider the corresponding short exact sequence

$$
1 \longrightarrow \operatorname{Ker}(\epsilon) \longrightarrow E \stackrel{\epsilon}{\longrightarrow} G \longrightarrow 1 \text {. }
$$

We say that the extension $(E, \epsilon)$ is split if the corresponding short exact sequence splits, i.e., there is a group morphism $\sigma$ such that $\epsilon \sigma=$ id. The morphism $\sigma$ itself is called a splitting.

Definition 9. A group $G$ is said to be centrally closed if $(G$,id) is a universal central extension of $G$.

A proof of the next fact can be found, e.g., in [2, Theorem 5.3].

Lemma 4. A central extension $(U, \pi)$ of a group $G$ is universal if and only if the group $U$ is perfect and every its central extension splits.

Using Lemma 4, we obtain the following result.

\footnotetext{
${ }^{1}$ Commutative diagrams are drawn using the package XY-pic.
} 
Corollary. A group $G$ is centrally closed if and only if it is perfect and every it central extension spits.

This corollary allows us to state Lemma 4 in the following form: a central extension $(U, \pi)$ is universal if and only if $U$ is centrally closed.

In the present paper it is shown that the unitary Steinberg group is centrally closed; therefore, as soon as the inclusion $\mathrm{K}_{2} \mathrm{U}(2 n, R, \Lambda) \subseteq \operatorname{Cent}(\operatorname{StU}(2 n, R, \Lambda))$ would be obtained under these or those assumptions, Theorem 1 of the present paper would imply immediately that under the same assumptions the unitary Steinberg group is a universal central extension of the elementary unitary group.

\section{§3. Plan of the proof of theorem 1}

The following commutator identities will be used substantially in our proofs. They can be checked by direct calculation.

Lemma 5. Let $G$ be a group; we denote by $[a, b]$ the commutator $a b a^{-1} b^{-1}$ of elements $a, b \in G$ and $b y{ }^{a} b$ the conjugate $a b a^{-1}$ of $b$ by $a$. Consider $x, y, z, y_{1}, \ldots, y_{n} \in G$. Then the following identities hold true.

$$
\begin{aligned}
& {[x y, z]={ }^{x}[y, z] \cdot[x, z]} \\
& {[x, y z]=[x, y] \cdot y[x, z]} \\
& {\left[x, y_{1} \cdot \ldots \cdot y_{n}\right]=\left[x, y_{1}\right] \cdot{ }^{y_{1}}\left[x, y_{2}\right] \cdot{ }^{y_{1} y_{2}}\left[x, y_{3}\right] \cdot \ldots \cdot{ }^{y_{1}} \cdot \ldots \cdot y_{n-1}\left[x, y_{n}\right]} \\
& {[x, y][x, z]=[x, y z][y,[z, x]]} \\
& { }^{y}\left[x,\left[y^{-1}, z\right]\right] \cdot{ }^{z}\left[y,\left[z^{-1}, x\right]\right] \cdot{ }^{x}\left[z,\left[x^{-1}, y\right]\right]=1 \quad \text { (the Hall-Witt identity), } \\
& z\left[y,\left[z^{-1}, x\right]\right]=\left[{ }^{z} y,[x, z]\right] .
\end{aligned}
$$

The next result follows from identities R4 and R6.

Lemma 6. The unitary Steinberg group $\operatorname{StU}(2 n, R, \Lambda)$ is perfect.

Thus, by the corollary to Lemma 4, to show that $\operatorname{StU}(2 n, R, \Lambda)$ is centrally closed it is necessary and sufficient to check that every central extension of it splits. In other words, Theorem 1 of the present paper follows directly from Theorem 2 .

In the proof of Theorem 2 we follow the plan of [2, Theorem 5.10], where it was proved that the linear Steinberg group is centrally closed. It is easier to describe the main idea of the proof in the notation of the linear case.

Let $\operatorname{GL}(n, R)$ be the general linear group over an associative unital ring, let $\mathrm{E}(n, R)$ be its elementary subgroup, and $\operatorname{St}(n, R)$ a Steinberg group defined by generators $x_{i j}(\zeta)$ and relations

$$
\begin{aligned}
& x_{i j}(\zeta) x_{i j}(\xi)=x_{i j}(\zeta+\xi), \\
& {\left[x_{i j}(\zeta), x_{h k}(\xi)\right]=1,} \\
& {\left[x_{i j}(\zeta), x_{j k}(\xi)\right]=x_{i k}(\zeta \xi),}
\end{aligned}
$$

with $i, j, h, k \in\{1, \ldots, n\}, j \neq h, i \neq k, \zeta, \xi \in R$. If a central extension $(E, \epsilon)$ of this group splits with $\sigma$, then in the inverse image of every generator $x_{i j}(\zeta)$ of the Steinberg group there is an element $s_{i j}(\zeta)=\sigma\left(x_{i j}(\zeta)\right)$ such that the Steinberg relations S1-S3 are fulfilled for $s_{i j}(\zeta)$. But the converse is also true, namely, if an element $s_{i j}(\zeta)$ in the inverse image of any generator is chosen such that the Steinberg relations are satisfied for them, then we can consider a natural morphism $\sigma: x_{i j}(\zeta) \mapsto s_{i j}(\zeta)$, which is obviously a splitting. Thus, to construct a splitting is the same as to find elements $s_{i j}(\zeta)$ as above.

Observe the following property of central extensions. 
Lemma 7. Let $(E, \epsilon)$ be a central extension of a group $G$. Then for any $g, h \in G$ and any $x_{1}, x_{2} \in \epsilon^{-1}(g), y_{1}, y_{2} \in \epsilon^{-1}(h)$, we have

$$
\left[x_{1}, y_{1}\right]=\left[x_{2}, y_{2}\right] \text {. }
$$

Proof. Since the extension $(E, \epsilon)$ is central, $x_{1} x_{2}^{-1}, y_{1} y_{2}^{-1} \in \operatorname{Ker}(\epsilon) \subseteq \operatorname{Cent}(E)$, so that $x_{1}=c x_{2}$ and $y_{1}=d y_{2}$ for appropriate $c, d \in \operatorname{Cent}(E)$, and $\left[x_{1}, y_{1}\right]=\left[c x_{2}, d y_{2}\right]=$ $\left[x_{2}, y_{2}\right]$.

This lemma leads us to the following definition.

Definition 10. Let $(E, \epsilon)$ be a central extension of $G$, and let $g, h \in G$. Then by $\left[\epsilon^{-1}(g), \epsilon^{-1}(h)\right]$ we denote the commutator $[x, y] \in E$ of any two elements $x \in \epsilon^{-1}(g)$ and $y \in \epsilon^{-1}(h)$.

Note that if the required elements $s_{i j}(\zeta)$ exist, then, in particular, relation S2 holds true for them:

$$
\left[s_{i j}(\zeta), s_{j k}(\xi)\right]=s_{i k}(\zeta \xi)
$$

which can be written in the form $s_{i k}(\zeta \xi)=\left[\epsilon^{-1} x_{i j}(\zeta), \epsilon^{-1} x_{j k}(\xi)\right]$, by using Lemma 7 . Thus, it is natural to define $s_{i k}(\zeta \xi)$ to be equal to $\left[\epsilon^{-1} x_{i j}(\zeta), \epsilon^{-1} x_{j k}(\xi)\right]$ (of course, we must check that the $s_{i k}(\zeta \xi)$ are well defined) and then show that the Steinberg relations are fulfilled for them, which allows us to extend the mapping $x_{i j}(\zeta) \mapsto s_{i j}(\zeta)$ up to a group morphism.

We return to the group $\mathrm{StU}(2 n, R, \Lambda)$ defined by the generators $X_{i j}(\zeta)$ and relations $\mathrm{R} 1-\mathrm{R} 6$. In the linear case we choose an element in the inverse image of a generator by using relation S2. Note that, for $\Lambda=\Lambda_{\text {min }}$, an element in the inverse image can be chosen by using either relation R4, or relation R5, and a substantial part of the proof can be extended easily from the linear case to this situation. For an arbitrary form parameter, relations R4 and R6 should be used. This plan is followed in $\S \S 4-5$.

\section{$\S 4$. Splitting is Well Defined as A Set Morphism}

In the sequel, $(E, \epsilon)$ denotes a central extension of $\operatorname{StU}(2 n, R, \Lambda)$ with $n \geq 5$.

First, we need the following lemma.

Lemma 8. Consider $i, j, h, k \in \Omega$ such that $i \neq j, h \neq k, h \neq j,-i, k \neq i,-j$. Then

$$
\left[\epsilon^{-1} X_{i j}(\zeta), \epsilon^{-1} X_{h k}(\xi)\right]=1 \text {. }
$$

Proof. First, let $h \neq-k$. Since $n \geq 5$, we can choose $l \neq \pm i, \pm j, \pm h, \pm k$ and consider $x \in \epsilon^{-1} X_{i j}(\zeta), y \in \epsilon^{-1} X_{h l}(\xi)$, and $z \in \epsilon^{-1} X_{l k}(1)$. Clearly, $[y, z] \in \epsilon^{-1} X_{h k}(\xi)$. Moreover, $[x, y],[x, z] \in \operatorname{Cent}(E)$. Then identity $\mathrm{C} 2$ implies

$$
1=\left[x, y^{-1} y\right]=\left[x, y^{-1}\right] \cdot y^{-1}[x, y]=\left[x, y^{-1}\right][x, y],
$$

i.e., $\left[x, y^{-1}\right]=[y, x]$, and, arguing as above, we get $\left[x, z^{-1}\right]=[z, x]$. Then, using C3 and the centrality of $[x, y]$ and $[x, z]$, we obtain

$$
\left[\epsilon^{-1} X_{i j}(\zeta), \epsilon^{-1} X_{h k}(\xi)\right]=[x,[y, z]]=[x, y][x, z]\left[x, y^{-1}\right]\left[x, z^{-1}\right]=1 .
$$

Now, let $h=-k$. Choose $l \neq \pm i, \pm j, \pm h$; then

$$
X_{h,-h}(\xi)=\left[X_{l,-l}\left(-\lambda^{(\varepsilon(h)-\varepsilon(l)) / 2} \xi\right), X_{-l,-h}(1)\right] X_{l,-h}\left(\lambda^{(\varepsilon(h)-\varepsilon(l)) / 2} \xi\right) .
$$

Consider $x \in \epsilon^{-1} X_{i j}(\zeta), u \in \epsilon^{-1} X_{l,-l}\left(-\lambda^{(\varepsilon(h)-\varepsilon(l)) / 2} \xi\right), v \in \epsilon^{-1} X_{-l,-h}(1)$, and

$$
z \in \epsilon^{-1} X_{l,-h}\left(\lambda^{(\varepsilon(h)-\varepsilon(l)) / 2} \xi\right) .
$$


It has already been shown that $[x, z]=1$. Clearly, $[x, u],[x, v] \in \operatorname{Cent}(E)$. Then, since $[u, v] z \in \epsilon^{-1} X_{h,-h}(\xi)$, relation $\mathrm{C} 2$ implies that

$$
\left[\epsilon^{-1} X_{i j}(\zeta), \epsilon^{-1} X_{h,-h}(\xi)\right]=[x,[u, v] z]=[x,[u, v]] \cdot{ }^{[u, v]}[x, z]=[x,[u, v]],
$$

but such a commutator is equal to the identity, as it was shown in the first part of the proof.

Lemma 8 claims that any inverse images $x$ and $y$ of the generators $X_{i j}(\zeta)$ and $X_{h k}(\xi)$ of the Steinberg group with $h \neq j,-i, k \neq i,-j$ commute. This fact will be used repeatedly in the sequel without special mention.

Now we proceed with the construction of a splitting. First, relation R4 leads us to the definition of $S_{h k}(\zeta)=\left[\epsilon^{-1} X_{h j}(\zeta), \epsilon^{-1} X_{j k}(1)\right] \in \epsilon^{-1} X_{h k}(\zeta)$ with $k \neq \pm h$. Still, we must check that this definition does not depend on the choice of $j$. In fact, a more general fact can be proved.

Lemma 9. Suppose $i, j, h, k \in \Omega, h \neq k, i, j \neq \pm h, \pm k, i \neq \pm j, \xi, \zeta \in R$. Then

$$
\left[\epsilon^{-1} X_{h i}(\zeta), \epsilon^{-1} X_{i k}(\xi)\right]=\left[\epsilon^{-1} X_{h j}(\zeta \xi), \epsilon^{-1} X_{j k}(1)\right] .
$$

Proof. Consider $x \in \epsilon^{-1} X_{h i}(\zeta), y \in \epsilon^{-1} X_{i j}(-\xi), z \in \epsilon^{-1} X_{j k}(1)$. Since $\left[z^{-1}, x\right]=1$, we can reformulate identity $\mathrm{C} 5$ as

$$
{ }^{y}\left[x,\left[y^{-1}, z\right]\right]={ }^{x}\left[\left[x^{-1}, y\right], z\right],
$$

but $\left[y^{-1}, z\right] \in \epsilon^{-1} X_{i k}(\xi)$ and $\left[x^{-1}, y\right] \in \epsilon^{-1} X_{h j}(\zeta \xi)$. For $k \neq-h$, we put $\eta=\zeta \xi$ and for $k=-h$, we put $\eta=\zeta \xi-\lambda^{-\varepsilon(h)} \bar{\xi}$. Now since $\left[x,\left[y^{-1}, z\right]\right]$ and $\left[\left[x^{-1}, y\right], z\right]$ belong to $\epsilon^{-1} X_{h k}(\eta)$, and thus, commute with $x$ and $y$, we arrive at the required identity.

Lemma 9 allows us to give the following definition.

Definition 11. Suppose $h, k \in \Omega, h \neq \pm k$, and $\zeta \in R$. We denote by $S_{h k}(\zeta)$ the element $\left[\epsilon^{-1} X_{h i}(\zeta), \epsilon^{-1} X_{i k}(1)\right]$ with $i \neq \pm h, \pm k$.

Note that Lemma 9 implies the following result.

Lemma 9.1. Suppose $j, h, k \in \Omega, h \neq \pm k, j \neq \pm h, \pm k, \xi, \zeta \in R$. Then

$$
\left[\epsilon^{-1} X_{h j}(\zeta), \epsilon^{-1} X_{j k}(\xi)\right]=S_{h k}(\zeta \xi) .
$$

While referring to Lemma 9 we shall sometimes mean Lemma 9A.

Clearly, for $\Lambda=\Lambda_{\min }$ Lemma 9 also allows us to define $S_{h k}(\alpha)$ with $h=-k$, $\alpha \in \lambda^{-(\varepsilon(h)+1) / 2} \Lambda_{\min }$, but for an arbitrary form parameter we must use R6 to define $S_{-k, k}(\alpha)$, i.e., to define this element by

$$
S_{-k, k}(\alpha)=\left[\epsilon^{-1} X_{j,-j}\left(-\lambda^{-(\varepsilon(j)+\varepsilon(k)) / 2} \alpha\right), \epsilon^{-1} X_{-j, k}(1)\right] S_{j k}\left(\lambda^{-(\varepsilon(j)+\varepsilon(k)) / 2} \alpha\right) .
$$

So, we must check that this expression does not depend on the choice of $j$; this follows from the next lemma.

Lemma 10. Suppose $i, j, k \in \Omega, i, j \neq \pm k, i \neq \pm j, \alpha \in \lambda^{(\varepsilon(k)-1) / 2} \Lambda, \xi \in R$. Then

$$
\begin{aligned}
& {\left[\epsilon^{-1} X_{i,-i}\left(-\lambda^{-(\varepsilon(i)+\varepsilon(k)) / 2} \bar{\xi} \alpha \xi\right), \epsilon^{-1} X_{-i, k}(1)\right] S_{i k}\left(\lambda^{-(\varepsilon(i)+\varepsilon(k)) / 2} \bar{\xi} \alpha \xi\right)} \\
& \quad=\left[\epsilon^{-1} X_{j,-j}\left(-\lambda^{-(\varepsilon(j)+\varepsilon(k)) / 2} \alpha\right), \epsilon^{-1} X_{-j, k}(\xi)\right] S_{j k}\left(\lambda^{-(\varepsilon(j)+\varepsilon(k)) / 2} \alpha \xi\right) .
\end{aligned}
$$

Proof. Consider $x \in \epsilon^{-1} X_{j,-j}\left(-\lambda^{-(\varepsilon(j)+\varepsilon(k)) / 2} \alpha\right), y \in \epsilon^{-1} X_{-j,-i}(-\xi)$,

$$
w \in \epsilon^{-1} X_{j,-i}\left(\lambda^{-(\varepsilon(j)+\varepsilon(k)) / 2} \alpha \xi\right),
$$

and $z \in \epsilon^{-1} X_{-i, k}(1)$. It is easily seen that

$$
\left[x^{-1}, y\right] w \in \epsilon^{-1} X_{i,-i}\left(-\lambda^{-(\varepsilon(i)+\varepsilon(k)) / 2} \bar{\xi} \alpha \xi\right),
$$


whence

$$
\begin{aligned}
& {\left[\epsilon^{-1} X_{i,-i}\left(-\lambda^{-(\varepsilon(i)+\varepsilon(k)) / 2} \bar{\xi} \alpha \xi\right), \epsilon^{-1} X_{-i, k}(1)\right]} \\
& \quad={ }^{x}\left[\epsilon^{-1} X_{i,-i}\left(-\lambda^{-(\varepsilon(i)+\varepsilon(k)) / 2} \bar{\xi} \alpha \xi\right), \epsilon^{-1} X_{-i, k}(1)\right]={ }^{x}\left[\left[x^{-1}, y\right] w, z\right] .
\end{aligned}
$$

Using $\mathrm{C} 1$, we can reshape this to

$$
{ }^{x}\left[\left[x^{-1}, y\right] \cdot w, z\right]={ }^{x\left[x^{-1}, y\right]}[w, z] \cdot{ }^{x}\left[\left[x^{-1}, y\right], z\right],
$$

and since $\left[z^{-1}, x\right]=1$, relation $\mathrm{C} 5$ shows that

$$
{ }^{x}\left[\left[x^{-1}, y\right], z\right]={ }^{y}\left[x,\left[y^{-1}, z\right]\right]=\left[y,\left[x,\left[y^{-1}, z\right]\right]\right] \cdot\left[x,\left[y^{-1}, z\right]\right] .
$$

Relation R1 in the Steinberg group implies $y \in \epsilon^{-1} X_{i j}\left(\lambda^{(\varepsilon(j)-\varepsilon(i)) / 2} \bar{\xi}\right)$. Moreover,

$$
\left[y^{-1}, z\right]=S_{-j, k}(\xi)
$$

whence

$$
\left[x,\left[y^{-1}, z\right]\right] \in \epsilon^{-1}\left(X_{j k}\left(-\lambda^{-(\varepsilon(j)+\varepsilon(k)) / 2} \alpha \xi\right) \cdot X_{-k, k}(\bar{\xi} \alpha \xi)\right) .
$$

Since $\left[\epsilon^{-1} X_{i j}\left(\lambda^{(\varepsilon(j)-\varepsilon(i)) / 2 \bar{\xi}}\right), \epsilon^{-1} X_{-k, k}(\bar{\xi} \alpha \xi)\right]=1$, from C2 it follows that

$$
\begin{aligned}
& {\left[y,\left[x,\left[y^{-1}, z\right]\right]\right]=\left[\epsilon^{-1} X_{i j}\left(\lambda^{(\varepsilon(j)-\varepsilon(i)) / 2} \bar{\xi}\right), \epsilon^{-1}\left(X_{j k}\left(-\lambda^{-(\varepsilon(j)+\varepsilon(k)) / 2} \alpha \xi\right) \cdot X_{-k, k}(\bar{\xi} \alpha \xi)\right)\right]} \\
& \quad=\left[\epsilon^{-1} X_{i j}\left(\lambda^{(\varepsilon(j)-\varepsilon(i)) / 2} \bar{\xi}\right), \epsilon^{-1} X_{j k}\left(-\lambda^{-(\varepsilon(j)+\varepsilon(k)) / 2} \alpha \xi\right)\right]=S_{i k}\left(-\lambda^{-(\varepsilon(i)+\varepsilon(k)) / 2} \bar{\xi} \alpha \xi\right) .
\end{aligned}
$$

Hence,

$$
{ }^{x}\left[\left[x^{-1}, y\right], z\right]=S_{i k}\left(-\lambda^{-(\varepsilon(i)+\varepsilon(k)) / 2} \bar{\xi} \alpha \xi\right)\left[\epsilon^{-1} X_{j,-j}\left(-\lambda^{-(\varepsilon(j)+\varepsilon(k)) / 2} \alpha\right), \epsilon^{1} X_{-j, k}(\xi)\right] .
$$

Now, using the fact that $[w, z]=S_{j k}\left(\lambda^{-(\varepsilon(j)+\varepsilon(k)) / 2} \alpha \xi\right)$,

$$
\left[x^{-1}, y\right] \in \epsilon^{-1}\left(X_{j,-i}\left(-\lambda^{-(\varepsilon(j)+\varepsilon(k)) / 2} \alpha \xi\right) \cdot X_{i,-i}\left(-\lambda^{-(\varepsilon(i)+\varepsilon(k)) / 2} \bar{\xi} \alpha \xi\right)\right),
$$

and $[w, z]$ commutes with both $\left[x^{-1}, y\right]$ and $x$, we obtain

$$
\begin{aligned}
x\left[\left[x^{-1}, y\right] \cdot w, z\right]=S_{j k}\left(\lambda^{-(\varepsilon(j)+\varepsilon(k)) / 2} \alpha \xi\right) & S_{i k}\left(-\lambda^{-(\varepsilon(i)+\varepsilon(k)) / 2} \bar{\xi} \alpha \xi\right) \\
& \times\left[\epsilon^{-1} X_{j,-j}\left(-\lambda^{-(\varepsilon(j)+\varepsilon(k)) / 2} \alpha\right), \epsilon^{1} X_{-j, k}(\xi)\right],
\end{aligned}
$$

which completes the proof.

Now we can give the following definition.

Definition 12. For $k \in \Omega, \alpha \in \lambda^{(\varepsilon(k)-1) / 2} \Lambda$, let $S_{-k, k}(\alpha)$ be the element

$$
\left[\epsilon^{-1} X_{i,-i}\left(-\lambda^{-(\varepsilon(i)+\varepsilon(k)) / 2} \alpha\right), \epsilon^{-1} X_{-i, k}(1)\right] S_{i k}\left(\lambda^{-(\varepsilon(i)+\varepsilon(k)) / 2} \alpha\right),
$$

with $i \neq \pm k$.

The above definition allows us to reformulate Lemma 10 in the following way.

Lemma 10.1. Suppose $j, k \in \Omega, j \neq \pm k, \alpha \in \Lambda, \xi \in R$. Then

$$
\left[\epsilon^{-1} X_{j,-j}\left(-\lambda^{-(\varepsilon(j)+\varepsilon(k)) / 2} \alpha\right), \epsilon^{-1} X_{-j, k}(\xi)\right] S_{j k}\left(\lambda^{-(\varepsilon(j)+\varepsilon(k)) / 2} \alpha \xi\right)=S_{-k, k}(\bar{\xi} \alpha \xi) .
$$

So, we have chosen an element $S_{i j}(\zeta)$ in the inverse image of every generator $X_{i j}(\zeta)$ of the Steinberg group. In the sequel we prove that the Steinberg relations R1-R6 are fulfilled for these $S_{i j}(\zeta)$. 


\section{$\S 5$. Splitting is Well Defined As A Group MORPhism}

In this section, $(E, \epsilon)$ denotes a central extension of the unitary Steinberg group $\operatorname{StU}(2 n, R, \Lambda)$ with $n \geq 5$, and the elements $S_{i j}(\zeta)$ are chosen as in Definitions 11 and 12, We show that the Steinberg relations R1-R6 are fulfilled for them.

First, we note that, since $S_{i j}(\zeta) \in \epsilon^{-1} X_{i j}(\zeta)$, from Lemmas 8, 9, and 10 it follows that relations R3, R4, and R6 hold true for $S_{i j}(\zeta)$, respectively. We check the remaining relations.

First, we deal with R2.

Lemma 11. Suppose that $i, j \in \Omega, i \neq j, \zeta, \xi \in R$, and that either $i \neq-j$, or $\zeta$, $\xi \in \lambda^{-(\varepsilon(i)+1) / 2} \Lambda$. Then

$$
S_{i j}(\zeta) S_{i j}(\xi)=S_{i j}(\zeta+\xi)
$$

Proof. First, let $i \neq-j$. Consider $l \neq \pm i, \pm j$ and $x \in \epsilon^{-1} X_{i l}(1), y \in \epsilon^{-1} X_{l j}(\zeta)$, $z \in \epsilon^{-1} X_{l j}(\xi)$. Then

$$
[x, y]=S_{i j}(\zeta), \quad[x, z]=S_{i j}(\xi), \quad[x, y z]=S_{i j}(\zeta+\xi)
$$

Moreover, $[y,[z, x]]=\left[\epsilon^{-1} X_{l j}(\zeta), \epsilon^{-1} X_{i j}(-\xi)\right]=1$. Thus, using C4, we get

$$
[x, y][x, z]=[x, y z]
$$

and the required identity follows.

Now, let $i=-j$. Suppose $k \neq \pm i, x \in \epsilon^{-1} X_{-i, k}(1), y \in \epsilon^{-1} X_{i,-i}\left(-\lambda^{-(\varepsilon(k)+\varepsilon(i)) / 2} \zeta\right)$, and $z \in \epsilon^{-1} X_{i,-i}\left(-\lambda^{-(\varepsilon(k)+\varepsilon(i)) / 2} \xi\right)$. We observe that

$$
[y,[z, x]]=\left[\epsilon^{-1} X_{i,-i}\left(-\lambda^{-(\varepsilon(k)+\varepsilon(i)) / 2} \zeta\right), \epsilon^{-1}\left(X_{i k}\left(-\lambda^{-(\varepsilon(k)+\varepsilon(i)) / 2} \xi\right) X_{-k, k}(\xi)\right)\right]=1 .
$$

Denote $S_{i k}\left(\lambda^{-(\varepsilon(k)+\varepsilon(i)) / 2} \zeta\right)$ by $u, S_{i k}\left(\lambda^{-(\varepsilon(k)+\varepsilon(i)) / 2} \xi\right)$ by $v$, and $S_{i k}\left(\lambda^{-(\varepsilon(k)+\varepsilon(i)) / 2}(\zeta+\xi)\right)$ by $w$. Above, it was shown that $u v=w$. Then identity $\mathrm{C} 4$ implies

$$
[z, x][y, x] u v=[y z, x] w,
$$

i.e.,

$$
\begin{aligned}
& {\left[\epsilon^{-1} X_{i,-i}\left(-\lambda^{-(\varepsilon(k)+\varepsilon(i)) / 2} \xi\right), \epsilon^{-1} X_{-i, k}(1)\right] S_{i k}\left(\lambda^{-(\varepsilon(k)+\varepsilon(i)) / 2} \xi\right)} \\
& \quad \times\left[\epsilon^{-1} X_{i,-i}\left(-\lambda^{-(\varepsilon(k)+\varepsilon(i)) / 2} \zeta\right), \epsilon^{-1} X_{-i, k}(1)\right] S_{i k}\left(\lambda^{-(\varepsilon(k)+\varepsilon(i)) / 2} \zeta\right) \\
& \quad=\left[\epsilon^{-1} X_{i,-i}\left(-\lambda^{-(\varepsilon(k)+\varepsilon(i)) / 2}(\xi+\zeta)\right), \epsilon^{-1} X_{-i, k}(1)\right] S_{i k}\left(\lambda^{-(\varepsilon(k)+\varepsilon(i)) / 2}(\xi+\zeta)\right),
\end{aligned}
$$

which completes the proof.

Now we check relation R1.

Lemma 12. Suppose that $i, j \in \Omega, i \neq j, \zeta \in R$ are such that either $i \neq-j$ or $\zeta \in \lambda^{-(\varepsilon(i)+1) / 2} \Lambda$. Then

$$
S_{i j}(\zeta)=S_{-j,-i}\left(-\lambda^{(\varepsilon(j)-\varepsilon(i)) / 2} \bar{\zeta}\right) .
$$

Proof. For $\zeta \in \lambda^{-(\varepsilon(i)+1) / 2} \Lambda$, the claim follows directly from the definition of the form parameter.

Now, let $i \neq-j$. Consider $l \neq \pm i, \pm j$. Using relation $\mathrm{R} 2$ already checked, we can complete the proof by the following calculation:

$$
\begin{aligned}
S_{i j}(\zeta) & =\left[\epsilon^{-1} X_{i l}(1), \epsilon^{-1} X_{l j}(\zeta)\right] \\
& =\left[\epsilon^{-1} X_{-l,-i}\left(-\lambda^{(\varepsilon(l)-\varepsilon(i)) / 2}\right), \epsilon^{-1} X_{-j,-l}\left(-\lambda^{(\varepsilon(j)-\varepsilon(l)) / 2} \bar{\zeta}\right)\right] \\
& =\left[\epsilon^{-1} X_{-j,-l}\left(-\lambda^{(\varepsilon(j)-\varepsilon(l)) / 2} \bar{\zeta}\right), \epsilon^{-1} X_{-l,-i}\left(-\lambda^{(\varepsilon(l)-\varepsilon(i)) / 2}\right)\right]^{-1} \\
& =S_{-j,-i}\left(\lambda^{(\varepsilon(j)-\varepsilon(i)) / 2} \bar{\zeta}\right)^{-1}=S_{-j,-i}\left(-\lambda^{(\varepsilon(j)-\varepsilon(i)) / 2} \bar{\zeta}\right) .
\end{aligned}
$$


We turn to the remaining relation R5. Lemma 9 allows us to prove a weaker statement.

Lemma 13. Suppose $j, k \in \Omega, j \neq \pm k$, and $\zeta \in R$. Then

$$
\left[\epsilon^{-1} X_{-k, j}(\zeta), \epsilon^{-1} X_{j k}(1)\right]=S_{-k, k}\left(\zeta-\lambda^{\varepsilon(k)} \bar{\zeta}\right) .
$$

Proof. For $l \neq \pm j, \pm k$, we denote $S_{j l}\left(-\lambda^{(\varepsilon(k)-\varepsilon(j)) / 2 \bar{\zeta})}\right.$ by $x, S_{l,-j}(1)$ by $y, S_{-j, k}(1)$ by $z$, and $S_{j k}\left(\lambda^{-(\varepsilon(j)+\varepsilon(k)) / 2} \zeta-\lambda^{(\varepsilon(k)-\varepsilon(j)) / 2} \bar{\zeta}\right)$ by $w$. Then

$$
S_{-k, k}\left(\zeta-\lambda^{\varepsilon(k)} \bar{\zeta}\right)={ }^{x} S_{-k, k}\left(\zeta-\lambda^{\varepsilon(k)} \bar{\zeta}\right)={ }^{x}\left[\left[x^{-1}, y\right], z\right] \cdot w .
$$

Using identity C5, we get

$$
{ }^{x}\left[\left[x^{-1}, y\right], z\right] \cdot w={ }^{y}\left[x,\left[y^{-1}, z\right]\right] \cdot{ }^{z}\left[y,\left[z^{-1}, x\right]\right] \cdot w .
$$

Since C1 implies

$$
{ }^{y}\left[y^{-1} \cdot{ }^{z} y,[x, z]\right]={ }^{y y^{-1}}\left[{ }^{z} y,[x, z]\right] \cdot{ }^{y}\left[y^{-1},[x, z]\right],
$$

and C6 implies

$$
{ }^{z}\left[y,\left[z^{-1}, x\right]\right]=\left[{ }^{z} y,[x, z]\right],
$$

it follows that

$$
{ }^{x}\left[\left[x^{-1}, y\right], z\right] \cdot w={ }^{y}\left[x,\left[y^{-1}, z\right]\right] \cdot{ }^{y}\left[\left[y^{-1}, z\right],[x, z]\right] \cdot{ }^{y}\left[[x, z], y^{-1}\right] \cdot w .
$$

It is easily seen that $\left[y^{-1}, z\right]=S_{l k}(-1)$ and $[x, z]=S_{-l, k}\left(\lambda^{(\varepsilon(l)-\varepsilon(k)) / 2} \zeta\right)$, whence

$$
\begin{aligned}
{\left[x,\left[y^{-1}, z\right]\right] } & =S_{j k}\left(\lambda^{(\varepsilon(k)-\varepsilon(j)) / 2} \bar{\zeta}\right), \\
{\left[[x, z], y^{-1}\right] } & =S_{j k}\left(-\lambda^{-(\varepsilon(j)+\varepsilon(k)) / 2} \zeta\right), \\
{\left[\left[y^{-1}, z\right],[x, z]\right] } & =\left[\epsilon^{-1} X_{-k,-l}\left(\lambda^{(\varepsilon(k)-\varepsilon(l)) / 2}\right), \epsilon^{-1} X_{-l, k}\left(\lambda^{(\varepsilon(l)-\varepsilon(k)) / 2} \zeta\right)\right] \\
& \in \epsilon^{-1} X_{-k, k}\left(\zeta-\lambda^{\varepsilon(k)} \bar{\zeta}\right),
\end{aligned}
$$

and all these elements commute with $y$ and one another. So, using the fact that

$$
\left[x,\left[y^{-1}, z\right]\right]\left[[x, z], y^{-1}\right]=w^{-1},
$$

we obtain

$$
\begin{aligned}
{ }^{x}\left[\left[x^{-1}, y\right], z\right] \cdot w & =\left[\left[y^{-1}, z\right],[x, z]\right] \\
& =\left[\epsilon^{-1} X_{-k,-l}\left(\lambda^{(\varepsilon(k)-\varepsilon(l)) / 2}\right), \epsilon^{-1} X_{-l, k}\left(\lambda^{(\varepsilon(l)-\varepsilon(k)) / 2} \zeta\right)\right] .
\end{aligned}
$$

To complete the proof, it remains to use Lemma 9 .

$$
\left[\epsilon^{-1} X_{-k,-l}\left(\lambda^{(\varepsilon(k)-\varepsilon(l)) / 2}\right), \epsilon^{-1} X_{-l, k}\left(\lambda^{(\varepsilon(l)-\varepsilon(k)) / 2} \zeta\right)\right]=\left[\epsilon^{-1} X_{-k, j}(\zeta), \epsilon^{-1} X_{j k}(1)\right] .
$$

Certainly, Lemma 13 immediately implies a more general result.

Lemma 13.1. Suppose $j, k \in \Omega, j \neq \pm k$, and $\zeta, \xi \in R$. Then

$$
\left[\epsilon^{-1} X_{-k, j}(\zeta), \epsilon^{-1} X_{j k}(\xi)\right]=S_{-k, k}\left(\zeta \xi-\lambda^{\varepsilon(k)} \overline{\xi \zeta}\right) .
$$

Proof. Suppose $l \neq \pm j, \pm k$. Using Lemmas 9 and 13, we have

$$
\left[\epsilon^{-1} X_{-k, j}(\zeta), \epsilon^{-1} X_{j k}(\xi)\right]=\left[\epsilon^{-1} X_{-k, l}(\zeta \xi), \epsilon^{-1} X_{l k}(1)\right]=S_{-k, k}\left(\zeta \xi-\lambda^{\varepsilon(k)} \overline{(\zeta \xi)}\right) .
$$

So, the Steinberg relations R1-R6 are fulfilled for $S_{i j}(\zeta)$, and we conclude that the unitary Steinberg group $\operatorname{StU}(2 n, R, \Lambda)$ is centrally closed. In the next section we formulate all the results obtained. 


\section{§6. Main Results}

Now we have all lemmas required to prove the claimed results. First, we prove Theorem 2 .

Proof of Theorem 2, Let $(E, \epsilon)$ be a central extension of the unitary Steinberg group, and let elements $S_{i j}(\zeta) \in E$ be as in Definitions 11 and 12. Then Lemmas 813 imply that the Steinberg relations R3, R4, R6, R2, R1, and R5 (respectively) hold true for $S_{i j}(\zeta)$. Thus, we can consider a natural group morphism $\sigma: \operatorname{StU}(2 n, R, \Lambda) \rightarrow E$ that sends the generator $X_{i j}(\zeta)$ to the element $S_{i j}(\zeta)$. Since $S_{i j}(\zeta) \in \epsilon^{-1} X_{i j}(\zeta)$, it follows that $\epsilon \sigma=\mathrm{id}$. Therefore, the extension $(E, \epsilon)$ splits.

Now Theorem 1 follows directly from the corollary to Lemma 4, Lemma 6, and Theorem 2 ,

In the case where the unitary Steinberg group in question is a central extension of the elementary unitary group, Theorem 1 implies the following statements.

Corollary 1. Let $n \geq 5$, and let $(R, \Lambda)$ be a form ring such that $(\operatorname{StU}(2 n, R, \Lambda), \varpi)$ is a central extension of the elementary unitary group $\mathrm{EU}(2 n, R, \Lambda)$. Then $(\operatorname{StU}(2 n, R, \Lambda), \varpi)$ is a universal central extension of $\mathrm{EU}(2 n, R, \Lambda)$.

Corollary 2. Let $n \geq 5$, and let $(R, \Lambda)$ be a form ring with $\mathrm{K}_{2} \mathrm{U}(2 n, R, \Lambda)$ central, i.e.,

$$
\mathrm{K}_{2} \mathrm{U}(2 n, R, \Lambda) \subseteq \operatorname{Cent}(\operatorname{StU}(2 n, R, \Lambda)) .
$$

Then $\mathrm{K}_{2} \mathrm{U}(2 n, R, \Lambda)$ coincides with the Schur multiplier of the elementary unitary group

$$
\mathrm{EU}(2 n, R, \Lambda) \text {. }
$$

Since the Schur multiplier of a group is precisely its second homology group with coefficients in integers, Corollary 2 can be reformulated as follows.

Corollary 3. Let $n \geq 5$, and let $(R, \Lambda)$ be a form ring with $\mathrm{K}_{2} \mathrm{U}(2 n, R, \Lambda)$ central. Then $\mathrm{K}_{2} \mathrm{U}(2 n, R, \Lambda)$ coincides with the second homology group of the elementary unitary group:

$$
\mathrm{K}_{2} \mathrm{U}(2 n, R, \Lambda)=\mathrm{H}_{2}(\mathrm{EU}(2 n, R, \Lambda), \mathbb{Z}) .
$$

Presently, the centrality of $\mathrm{K}_{2}$ is proved only in the linear case for arbitrary rings finite over its center (see [6, 15]). A similar result for classical even groups (in particular, for the even unitary group discussed in the present paper) was announced by Anthony Bak and Guoping Tang, still the detailed proof has not appeared so far.

\section{CONCLUSION}

In this paper it is proved that the even unitary Steinberg group $\operatorname{StU}(2 n, R, \Lambda)$ defined by Bak is centrally closed. The author plans to extend this result to the case of odd unitary groups, as defined by Viktor Petrov. At present, the last groups are the most wide and natural generalization of the even unitary groups described in this paper. Some information about odd unitary groups can be found in [3, 4.

I would like to express my thanks to my adviser Prof. Nikolai Vavilov and also to Prof. Anthony Bak communication with whom allowed me to deeper understand the current results and methods of the unitary $K$-theory.

\section{REFERENCES}

[1] E. V. Dybkova, Subgroups of hyperbolic unitary groups, Dokt. diss., S.-Peterburg. Gos. Univ., St. Petersburg, 2006. (Russian)

[2] J. Milnor, Introduction to algebraic K-theory, Ann. of Math. Stud., No. 72, Princeton Univ. Press, Princeton, NJ, 1971. MR0349811 (50:2304) 
[3] V. A. Petrov, Odd unitary groups, Zap. Nauchn. Sem. S.-Peterburg. Otdel. Mat. Inst. Steklov. (POMI) 305 (2003), 195-225; English transl., J. Math. Sci. (N. Y.) 130 (2005), no. 3, 4752-4766. MR2033642(2005b:20100)

[4] , Overgroups of classical groups, Kand. diss., S.-Peterburg. Gos. Univ., St. Petersburg, 2005. (Russian)

[5] R. Steinberg, Lectures on Chevalley groups, Yale Univ., New Haven, Conn., 1968. MR0466335 $(57: 6215)$

[6] M. S. Tulenbaev, The Schur multiplier of the group of elementary matrices of finite order, Zap. Nauchn. Sem. Leningrad. Otdel. Mat. Inst. Steklov. (LOMI) 86 (1979), 162-169; English transl. in J. Soviet Math. 17 (1981), no. 4. MR535488 (80j:18019)

[7] A. Bak, The stable structure of quadratic modules, Thesis Columbia Univ., 1969.

[8] _ On modules with quadratic forms, Algebraic K-Theory and Its Geometric Applications (Hull, 1969), Springer-Verlag, Berlin, 1969, pp. 55-66. MR0252431(40:5651)

[9] _ K-theory of forms, Ann. of Math. Stud., vol. 98, Princeton Univ. Press, Princeton, NJ, 1981. MR0632404 (84m:10012)

[10] A. Bak and N. Vavilov, Structure of hyperbolic unitary groups. I. Elementary subgroups, Algebra Colloq. 7 (2000), no. 2, 159-196. MR1810843 (2002b:20070)

[11] A. Hahn and O. O'Meara, The classical groups and K-theory, Grundlehren Math. Wiss., Bd. 291, Springer-Verlag, Berlin, 1989. MR 1007302 (90i:20002)

[12] R. Hazrat, Dimension theory and nonstable $\mathrm{K}_{1}$ of quadratic modules, K-Theory 27 (2002), 293-328. MR.1962906 (2004a:19005)

[13] R. Hazrat and N. Vavilov, Bak's work on K-theory of rings, with an appendix by Max Karoubi, J. K-Theory 4 (2009), 1-65. MR2538715 (2011d:19001)

[14] R. Hazrat, N. Vavilov, and Zuhong Zhang, Relative unitary commutator calculus, and applications, J. Algebra 343 (2011), 107-137. MR2824547(2012g:20093)

[15] W. van der Kallen, Another presentation for Steinberg groups, Indag. Math. 39 (1977), 304-312. MR 0463263(57:3216)

[16] M. Stein, Generators, relations and coverings of Chevalley groups over commutative rings, Amer. J. Math. 93 (1971), 965-1004. MR0322073 (48:437)

[17] A. Stepanov and N. Vavilov, Decomposition of transvections: a theme with variations, K-Theory 19 (2000), 109-153. MR1740757 (2000m:20076)

[18] Guoping Tang, Hermitian groups and K-theory, K-Theory 13 (1998), 209-267. MR.1609905 (99b:19006)

Department of mathematics and mechanics, St. Petersburg State University, UniversitetSkil̆ Pr. 28, Staryi Peterhof, St. Petersburg 198504, Russia

E-mail address: avlavrenov@gmail.com

Received 22/MAY/2012

Translated by THE AUTHOR 